\title{
Structure Calculations Applied to Shape Memory Alloys
}

\author{
Y. Gillet, E. Patoor and M. Berveiller \\ Laboratoire de Physique et Mécanique des Matériaux (UAR CNRS 1215), Institut Supérieur de Génie Mé- \\ canique et Productique, Université de Metz, Ile du Saulcy, 57045 Metz cedex, France
}

\begin{abstract}
Despite the potential of these alloys and the amount of patents, successful industrial and commercial applications of Shape Memory Alloys (SMA) remain exceptions. This can be explained by the lack of design tools able to dimension correctly SMA elements because the non elastic behavior of SMA lead the classical method of design to be inefficient. Furthermore, this behavior is strongly dependent on temperature and exhibits hysteresis. From the definition of constitutive equations describing the superelastic behavior for an homogeneous stress field, we derive a scheme of resolution for mechanical devices. Results obtained by this way are compared with experimental observations performed on $\mathrm{Cu}$ - based alloys.
\end{abstract}

\section{INTRODUCTION}

Even if a lot of study were conducted to describe the behavior of SMA, very few work [1], [2] have been done considering these alloys as mechanical elements where the stress distribution is, in many cases, inhomogeneous. In this work, structure calculations are performed with these materials. The determination of the load-displacement response of a structure requires the solution of two different problems. The first one is the definition of the behavior characterizing the material. The second one is the structure calculation that is related to geometrical considerations.

At first a modeling of the behavior of SMA is developed. The non symmetry between tensile and compressive tests observed experimentally is also taken into account through the definition of a non symmetric transformation criterion. From this criterion, an associated flow rule is obtained to describe the SMA behavior. Hysteretic effects are accounted introducing a kinematical hardening parameter. Considering the geometry of the most common elements made of SMA, structure calculation is performed into the framework of beam theory. To determine the response of SMA structures, an incremental formulation of Bresse integrals is used taking account of the coexistence of transforming zones and elastic ones during the loading and the unloading process. The results obtained by this way are successfully compared with experimental ones performed on superelastic springs and beams made of copper based SMA. Material parameters required for the computation are experimentally determined using tensile test and calorimetric measurements. An extension to thermomechanical loading is also presented in this study.

\section{CONSTITUTIVE EQUATIONS}

\subsection{Transformation criterion}

The particular characteristics of the SMA behavior impose to use a non symmetrical transformation criterion [3], [4], [5] since the behavior of the material is different in tension and in compression. 


$$
\mathrm{F}\left(\mathrm{J}_{2}, \mathrm{~J}_{3}, \mathrm{~T}, \varepsilon^{\mathrm{pt}}\right)=\mathrm{J}_{2}\left(1+\frac{\mathrm{b} \mathrm{J}_{3}}{\mathrm{~J}_{2}{ }^{3 / 2}}\right)-\mathrm{K}^{2}\left(\varepsilon^{\mathrm{pt}}, \mathrm{T}\right)=0
$$

in which $\mathrm{J}_{2}$ and $\mathrm{J}_{3}$ are respectively the second and the third invariant of the deviatoric part of the stress tensor $\sigma$. This expression is derived from the Prager equation $\left({ }^{1}\right)$. Its use is justified by micromechanical considerations [1]. Assuming infinitesimal strain theory, an increment of the total strain, denoted $d \varepsilon_{\mathrm{ij}}$, can be divided into an elastic part $\left(\mathrm{d} \varepsilon_{\mathrm{ij}}^{\mathrm{e}}\right)$, a thermal part $\left(\mathrm{d} \varepsilon_{\mathrm{ij}}^{\mathrm{th}}\right)$ and a transformation part $\left(\mathrm{d} \varepsilon_{\mathrm{ij}}^{\mathrm{pt}}\right)$. In analogy with associated plasticity, we choose $\varepsilon$ pt, the transformation strain, as an internal variable. The associated flow rule is obtained from the normality rule:

$$
\mathrm{d} \varepsilon_{\mathrm{ij}}^{\mathrm{pt}}=\mathrm{d} \lambda \cdot \frac{\partial \mathrm{F}}{\partial \sigma_{\mathrm{ij}}}
$$

The plastic multiplier is derived from the consistency condition:

$$
\mathrm{dF}=0=\frac{\partial \mathrm{F}}{\partial \sigma_{i j}} \mathrm{~d} \sigma_{\mathrm{ij}}+\frac{\partial \mathrm{F}}{\partial \varepsilon^{\mathrm{pt}}} \mathrm{d} \varepsilon^{\mathrm{pt}}+\frac{\partial \mathrm{F}}{\partial \mathrm{T}} \mathrm{dT}
$$

This defines a constitutive equation that can be used in structure calculations [4].

$$
\mathrm{d} \varepsilon_{1 j}^{\mathrm{pt}}=\frac{\partial \mathrm{F}}{\partial \sigma_{\mathrm{ij}}}\left(\frac{\frac{\partial \mathrm{F}}{\partial \sigma_{\mathrm{kl}}} \mathrm{d} \sigma_{\mathrm{kl}}-2 \mathrm{~K} \frac{\partial \mathrm{K}}{\partial \mathrm{T}} \mathrm{dT}}{2 . \mathrm{K} \cdot \frac{\partial \mathrm{K}}{\partial \varepsilon^{\mathrm{pt}}} \sqrt{\frac{2}{3} \frac{\partial \mathrm{F}}{\partial \sigma_{\mathrm{pq}}} \frac{\partial \mathrm{F}}{\partial \sigma_{\mathrm{pq}}}}}\right) \text { with } \frac{\partial \mathrm{F}}{\partial \sigma_{\mathrm{ij}}}=\frac{\partial \mathrm{J}_{2}}{\partial \sigma_{\mathrm{ij}}}-\frac{\mathrm{b}}{\mathrm{J}_{2}{ }^{3 / 2}}\left(\mathrm{~J}_{3} \cdot \frac{\partial \mathrm{J}_{2}}{\partial \sigma_{\mathrm{ij}}}-2 \cdot \mathrm{J}_{2} \cdot \frac{\partial \mathrm{J}_{3}}{\partial \sigma_{\mathrm{ij}}}\right)
$$

The criterion is identified using tensile/compression test or numerical results from a micromechanical approach [2]. The different parameters inherent to the material behavior are determined from measurements on particular testing having an homogeneous stress field:

$$
\mathrm{b}=\frac{\sqrt{27}}{2} \frac{\sigma_{\mathrm{c}}^{2}-\sigma_{\mathrm{t}}^{2}}{\sigma_{\mathrm{c}}^{2}+\sigma_{\mathrm{t}}^{2}} \quad \text { and } \quad \mathrm{K}\left(\varepsilon^{\mathrm{pt}}, \mathrm{T}\right)=\frac{\sigma}{\sqrt{3}} \sqrt{1+\frac{2 \mathrm{~b}}{\sqrt{27}}}
$$

In which $\sigma_{\mathrm{c}}$ and $\sigma_{\mathrm{t}}$ are the stress respectively in compression and tension.

The partial derivatives of function $\mathrm{K}\left(\varepsilon^{\mathrm{pt}}, \mathrm{T}\right)$ can be then calculated by choosing the expression of the stress as a parabolic approximation, as it is done in classical plasticity:

$$
\sigma=\sigma_{\mathrm{t}}=\sigma_{0}(\mathrm{~T})+\mathrm{K}_{0}\left(\varepsilon^{\mathrm{pt}}\right)^{\mathrm{n} 0}
$$

with

$$
\sigma_{0}(T)=B_{0}\left(T-M_{S}\right)
$$

where $B_{0}$ is a coefficient of proportionality between stress and temperature, and $\mathrm{K}_{0}, \mathrm{n}_{0}$ represent a transformation hardening. Figure 1 gives an example of comparison between experimental results obtained on a $\mathrm{Cu}$ based alloy and this approximation.

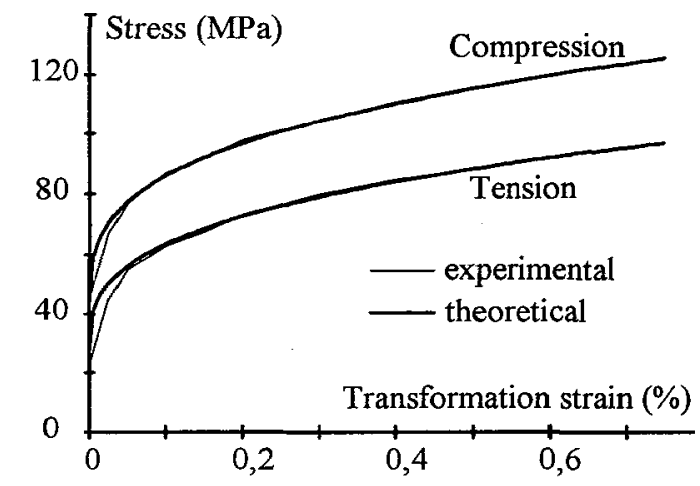

Figure 1: Tensile-compressive test curves obtained on a $\mathrm{Cu}-\mathrm{Zn}-\mathrm{Al}$ alloy with $\mathrm{Ms}=0^{\circ} \mathrm{C}$ [6] compared to the calculated one with a parabolic approximation.

$\left.{ }^{1}\right)$ note : the Von Mises symmetrical criterion is obtained with $b=0$. 


\subsection{Hysteresis}

Considering the unloading, a similar approach is done. The assumption of proportional loading is made. To describe the hysteresis, a kinematical hardening parameter denoted by $\mathrm{X}$ is introduced.

$$
X_{i j}=\left(\sigma_{0 i j}-\sigma_{f_{i j}}\left[\frac{\varepsilon}{\varepsilon^{*}}\left(1-\frac{\varepsilon^{*}}{\varepsilon_{a m}}\right)-1\right]\right.
$$

The reverse transformation criterion is then expressed by:

$$
F_{\mathrm{d}}\left(\mathrm{J}_{2}^{\prime}, \mathrm{J}_{3}^{\prime}, \varepsilon^{\mathrm{pt}}, \mathrm{X}, \mathrm{T}\right)=\mathrm{J}_{2}^{\prime}\left(1+\frac{\mathrm{b} \mathrm{J}_{3}^{\prime}}{\mathrm{J}_{2}{ }^{3} \dot{2}}\right)-\mathrm{K}^{2}\left(\varepsilon^{\mathrm{pt}}, \mathrm{T}\right)=0
$$

where $J_{2}^{\prime}$ and $J_{3}^{\prime}$ are respectively the second and the third invariant of $(\sigma-X)$. The superelastic behavior described in that way is summarized on the following curve (fig. 2) and in table 1.

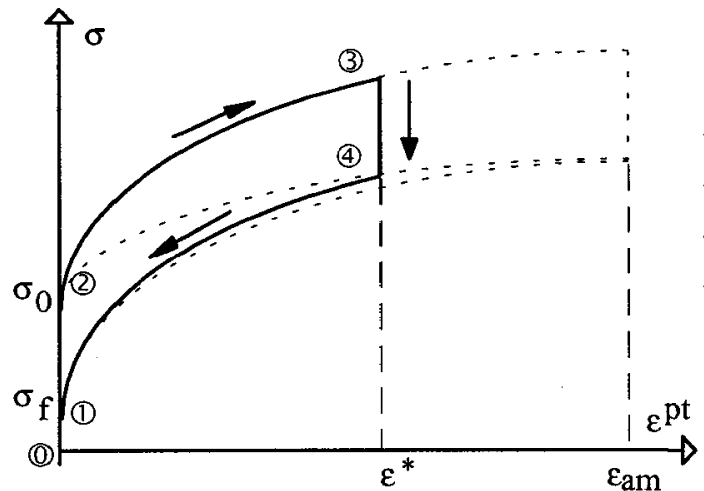

Figure 2: Diagram of principle

\begin{tabular}{|c|c|c|c|c|l|}
\cline { 2 - 5 } \multicolumn{1}{c|}{} & $\mathrm{F}$ & $\dot{\mathrm{F}}$ & $\mathrm{F}_{\mathbf{d}}$ & $\dot{\mathrm{F}}_{\mathrm{d}}$ & \\
\hline (1) (1) & $<0$ & & $<0$ & & elastic behavior \\
\hline at (1) & $<0$ & & $=0$ & $\neq 0$ & elastic behavior \\
\hline (1) (2) & $<0$ & & $>0$ & & elastic behavior \\
\hline (2) (3) & $=\mathbf{0}$ & $=\mathbf{0}$ & $>0$ & & $\Rightarrow \quad \mathbf{d \varepsilon p t > 0}$ \\
\hline at (3) & $=\mathbf{0}$ & $<\mathbf{0}$ & & & $\Rightarrow \quad \mathbf{d \varepsilon p t = 0}$ \\
\hline (3) (4) & $<0$ & & $>0$ & & elastic behavior \\
\hline (4) (1) & $<0$ & & $=\mathbf{0}$ & $=\mathbf{0}$ & $\Rightarrow \quad \mathbf{d \varepsilon p t}<\mathbf{0}$ \\
\hline
\end{tabular}

Table 1: Values of the criteria and their derivatives along a superelastic cycle

This behavior is now used in structure calculation taking $\mathrm{n}_{0}$ equal to one (bilinear approximation).

\section{SUPERELASTIC BEAMS}

\subsection{Displacements and rotations} relation 11 ).

The displacements and the rotations of the structure are given by the Bresse integrals method [7]. Displacement of section $B$ is a function of the displacement and rotation in $\mathrm{A}$ and the contribution of elementary displacements and rotations between $A$ and $B$ (see relation 10). Rotation of section B is a function of the rotation in $A$ and the contribution of elementary rotations between A and B (see

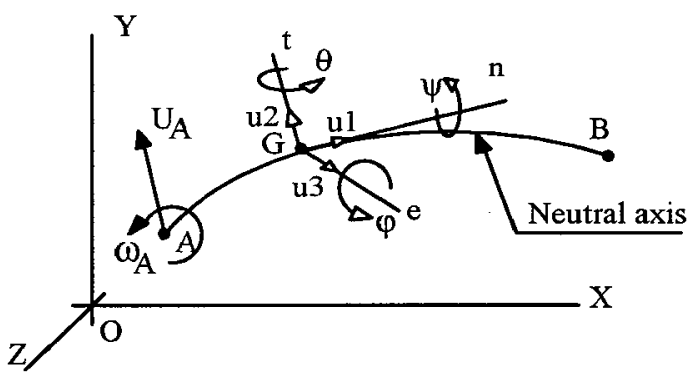

Figure 3: Description of displacements and rotations using Bresse method.

$$
\overrightarrow{\mathrm{U}}_{\mathrm{B}}=\overrightarrow{\mathrm{U}}_{\mathrm{A}}+\vec{\omega}_{\mathrm{A}} \wedge \overrightarrow{\mathrm{AB}}+\int_{\widehat{A B}}(\mathrm{u} 1 \overrightarrow{\mathrm{n}}+\mathrm{u} 2 \overrightarrow{\mathrm{t}}+\mathrm{u} 3 \overrightarrow{\mathrm{e}}) \mathrm{ds} \underset{\widehat{\mathrm{AB}}}{\int_{\widehat{A}}}(\psi \overrightarrow{\mathrm{n}}+\theta \overrightarrow{\mathrm{t}}+\varphi \overrightarrow{\mathrm{e}}) \wedge \overrightarrow{\mathrm{GB}} \mathrm{ds}
$$




$$
\vec{\omega}_{B}=\vec{\omega}_{A}+\int_{A}(\psi \vec{n}+\theta \vec{t}+\varphi \vec{e}) d s
$$

The elementary rotations and displacements are function of the internal forces, the geometry of the section, and the material parameters. In the case of SMA, the different integrals present in (12) are not constant and vary with the transformation.

$$
\left\{\begin{array}{ccc}
u_{1}=\frac{N}{\int_{S} E d S} & u_{2}=\frac{T_{2}}{\int_{S} G d S} & u_{3}=\frac{T_{3}}{\int_{S} G d S} \\
\psi=\frac{M_{t}}{\int_{S} G \rho^{2} d S} & \theta=\frac{M_{f}}{\int_{S} E z^{2} d S} & \varphi=\frac{M_{f 3}}{\int_{S} E y^{2} d S}
\end{array}\right.
$$

Due to the geometry of elements and non homogeneous strain field in the material, there is coexistence of untransformed zones (austenite), transforming zones, transformed zones (martensite).

\subsection{Pure torsion of a cylindrical rod}

In this case, the transformation proceeds from the outer diameter, where the fibers are the most loaded, to the center of the circular section. The center of the section remains elastic while the transforming or transformed zones are concentric rings [8], [9]. Helical springs can be calculated if the wire is considered submitted only to pure torsion. An example is given on the following curves for a superelastic spring. The material parameters needed for the modeling are deduced from tensile test (fig. 4) while the comparison between theoretical results and experimental ones are given in fig. 5 .

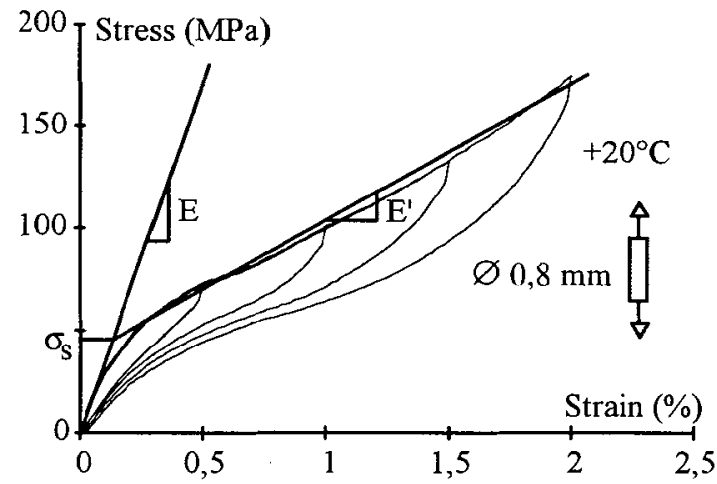

Figure 4: Tensile test curve performed on a Cu-Zn-Al-Ni alloy at ambient temperature (wire $\varnothing 0,8 \mathrm{~mm}$ )

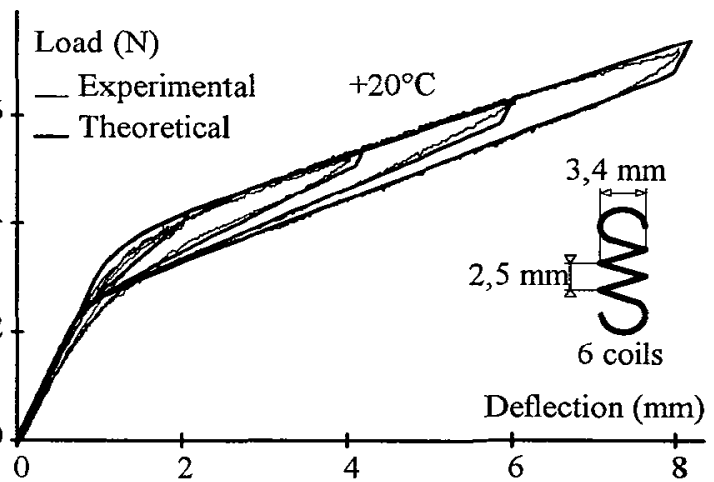

Figure 5: Load-displacement curve of an helical spring performed on the same alloy compared to the calculated curve (wire $\varnothing 0,8 \mathrm{~mm}$ ).

\subsection{Bending of beams}

In general cases of bending, internal forces depend on the position of the section. It is then necessary to discretize the beam length. Furthermore, the geometry must be reactualized after each calculation step because large displacements of the beam available in bending. And finally, the non symmetric transformation of the cross section (see figure 6) due to the non symmetry between tension and compression lead to a shift of the neutral axis that have to be accounted. 


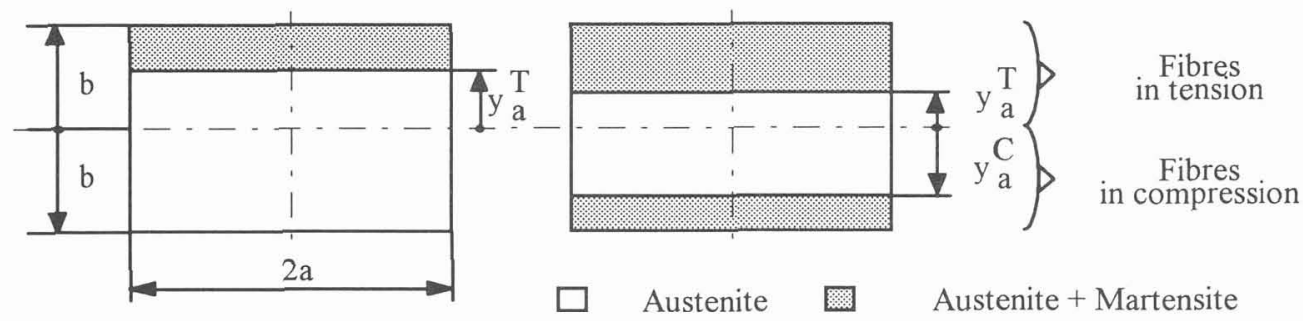

Figure 6: Evolution of the transformed zones in bending

Results obtained for three point bending on a superelastic beam are presented on the following. The different parameters of the material are obtained from the tensile test curve shown in fig. 7 and micromechanical results. The comparison between theoretical and experimental results is made in fig. 8 .

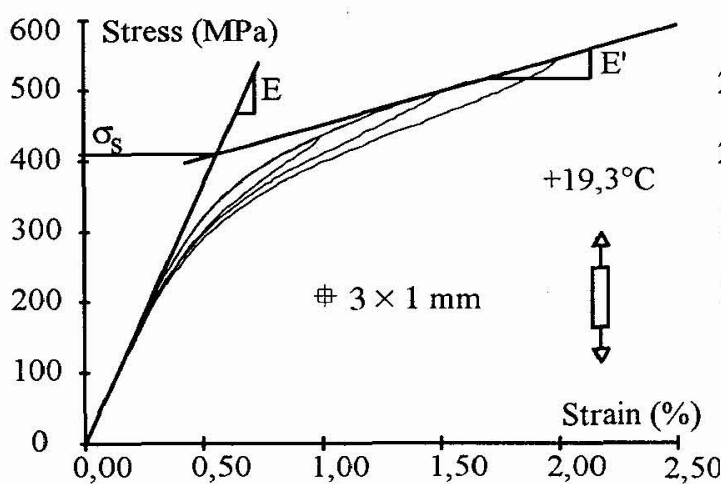

Figure 7: Tensile test curve performed on a Cu-Al-Be alloy

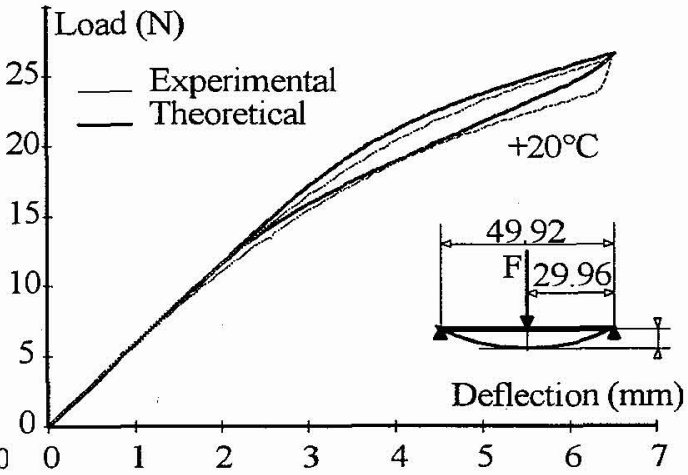

Figure 8: Load-displacement curve obtained on a three point bending test compared to the calculated one.

These two examples of structure calculation show good agreement between experimental and theoretical results.

\section{THERMOMECHANICAL LOADING}

In this case, some restrictive assumptions have been made concerning the material behavior. At first the material is assumed to be cooled from the austenitic state and heated until the austenitic state is reached. The assumption of proportional loading is conserved and the external loading applied to the structure can not reach zero.

In first approximation the thermal hysteresis is neglected (i.e. $M_{S}(0)=A_{f}(0)$ and $\left.M_{f}(0)=A_{S}(0)\right)$. It is also considered that there is no more deformation above $\mathrm{M}_{\mathrm{s}}(0)$ if $\mathrm{M}_{\mathrm{s}}(0)$ is greater $\mathrm{M}_{\mathrm{f}}(\sigma)$. Some theoretical results are presented below for two type of thermomechanical loading applied to an helical spring. Tendencies experimentally observed are qualitatively found on these curves despite the large assumptions made.

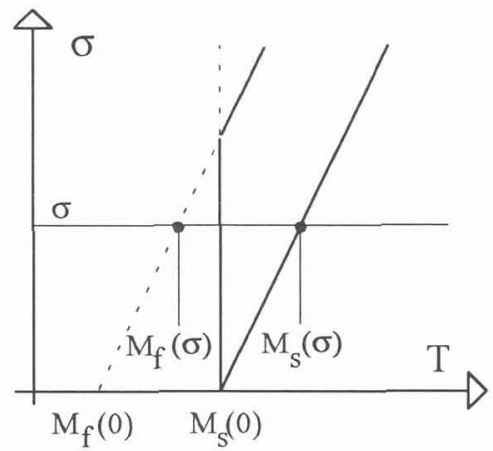

Figure 9: State diagram in first approximation. 


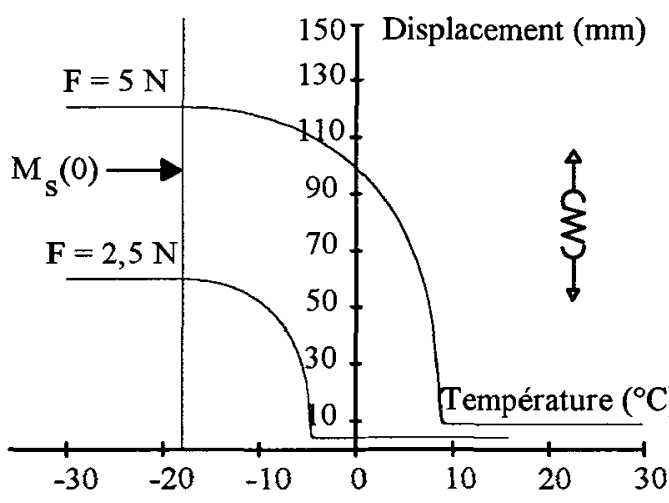

Figure 10: Cooling of a spring under a constant load.

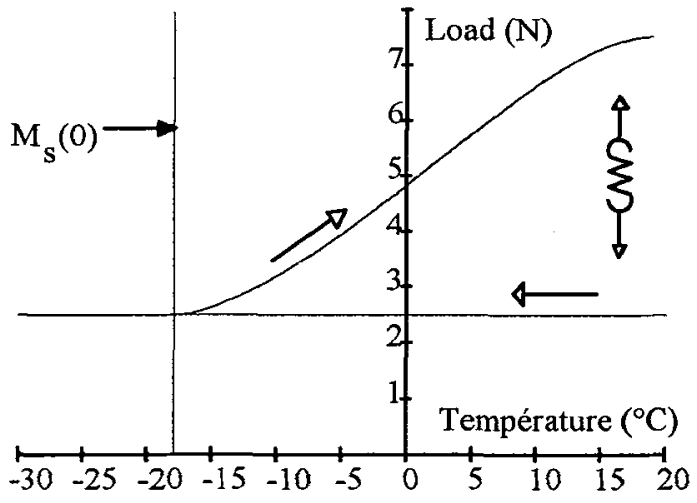

Figure 11: Cooling of the same spring under a constant load then heating keeping the displacement constant.

\section{CONCLUSION}

Results obtained in superelasticity are in good agreement with experimental ones. Reliable design tools have been developed and give rapidly results on computers of PC type. Concerning thermomechanical loading some more efforts have to be done to describe the SMA behavior below $\mathrm{M}_{\mathrm{S}}$. The introduction of the thermal hysteresis is necessary to describe correctly the memory effect and a particular attention must be kept on the problem of reorientation of martensite. Even though, some qualitative results are obtained with a very simplified behavior.

\section{References}

[1] TOBUSHI H. et TANAKA K., "Deformation of a Shape Memory Alloy Helical Spring.", JSME Int. Journal, 1991, Series 1, Vol. 34, No. 1, p. 83.

[2] ATANACKOVIC T, ACHENBACH M., "Moment-curvature relations for a pseudoelastic beam", Continuum Mech. Thermodyn., 1989, p.73.

[3] EL AMRANI ZIRIFI M., "Contributions à l'étude micromécanique des transformations martensitiques thermoélastiques", Thèse de doctorat, Université de Metz, 1994.

[4] GILLET Y., "Dimensionnement d'éléments simples en alliage à mémoire de forme", Thèse de doctorat, Université de Metz, 1994.

[5] E. PATOOR, M. EL AMRANI, A. EBERHARDT, M. BERVEILLER, "Determination of the origin for the dissymetry observed between tensile and compression tests on shape memory alloys", This conference.

[6] VACHER P., "Étude du comportement pseudoélastique d'alliagesà mémoire de forme $\mathrm{Cu}-\mathrm{Zn}-\mathrm{Al}$ polycristallins", Thèse de doctorat $\mathrm{N}^{\circ} 215$, Université de Franche-Comté, Besançon, 1991.

[7] BRESSE J.M., Cours de mécanique appliquée - Première partie: Résistance des matériaux et stabilité des constructions, Paris, 1880.

[8] GILLET Y., PATOOR E., BERVEILLER M., "Mechanical engineering for shape memory alloys", ICOMAT 92, Monterey (USA), 1992, p.

[9] GILLET Y., PATOOR E., BERVEILLER M., "Beam theory applied to shape memory alloys", SMST '94, Monterey, 1994, To be published. 